\title{
LA-UR-21-24617
}

Approved for public release; distribution is unlimited.

Title: $\quad$ Large Eddy Simulations of Turbulence below Antarctic Ice Shelves

Author(s): $\quad$ Asay-Davis, Xylar Storm

Begeman, Carolyn Branecky

Van Roekel, Luke

Intended for: Report

Issued: 
Disclaimer:

Los Alamos National Laboratory, an affirmative action/equal opportunity employer, is operated by Triad National Security, LLC for the National Nuclear Security Administration of U.S. Department of Energy under contract 89233218CNA000001. By approving this article, the publisher recognizes that the U.S. Government retains nonexclusive, royalty-free license to publish or reproduce the published form of this contribution, or to allow others to do so, for U.S. Government purposes. Los Alamos National Laboratory requests that the publisher identify this article as work performed under the auspices of the U.S. Department of Energy. Los Alamos National Laboratory strongly supports academic freedom and a researcher's right to publish; as an institution, however, the Laboratory does not endorse the viewpoint of a publication or guarantee its technical correctness. 


\section{Large Eddy Simulations of Turbulence below Antarctic Ice Shelves}

Xylar Asay-Davis, Carolyn Begeman, Luke Van Roekel, Stephen Price

\section{Scientific and programmatic impact}

Turbulence below ice shelves is of key importance for predicting ice-shelf melt rates and consequently the contribution of ice sheets to sea level rise. In this project we conducted Large-Eddy Simulations (LES) to improve our understanding of sub-ice-shelf ocean turbulence and the relationship between ice-shelf melt rates and ocean conditions. Over the course of the second and final year of this project (FY2020), we accomplished both major code developments for the PArallel Large-eddy simulation Model (PALM; Maronga et al., 2015) and conducted a suite of simulations which form the basis for a publication in preparation.

Many modifications to PALM were needed to provide a suitable configuration and dynamics for sub-ice settings. These developments benefitted not only this project but also the ongoing LANL-led project InterFACE, a component of which addresses turbulence below sea-ice with LES. Most of the model development needed to represent ice ablating into seawater and to support a sub-ice configuration were completed in Year 1. The main model development tasks we accomplished in Year 2 were the addition of output variables needed to compute energy budgets and the implementation of a state-of-the-art turbulence closure scheme, Anisotropic Minimum Dissipation (Abkar et al., 2016). One of the scientific findings of this study that motivated the latter implementation was excess dissipation of energy using traditional turbulence closure schemes.

This project yielded novel scientific insights into stratified, wall-bounded turbulence in the presence of an ablating, sloped boundary. These conditions had not previously been studied at the intermediate (10s to 100 s meters) scale of our large-eddy simulations, and this work was only recently made possible by finer-scale studies (Direct Numerical Simulations and laboratory experiments) that shed light on ice-ocean interface processes unresolved by our simulations. This puts LANL at the forefront of the study of ice-ocean interactions, which has garnered momentum due to the rapid changes in sea-ice and ice-shelf thickness in recent decades.

Our key simulations comprise a parameter study of (a) the effect of varying far-field ocean temperature and (b) the effect of varying ice-shelf slope. The primary scientific objective of our study is the improvement of ice-shelf melt parameterizations used in coarse-resolution ocean models such as the ocean model component of the DOE Energy Exascale Earth System Model (E3SM). Our simulated relationship between far-field ocean temperature and melt rates agrees with commonly-used parameterizations. On the other hand, we find that even very low ice-shelf slopes characteristic of Antarctic settings affect the flow dynamics with consequences for melt parameterizations. Our simulations also raise the possibility that slope orientation relative to background pressure gradients affects melt rates. This finding deserves further study since melt parameterizations depend on flow magnitude but not orientation. 
The translation of these scientific findings into an improved ice-shelf melt parameterization for the ocean component of E3SM has proven to be more difficult than originally conceived due to the tendency for highly stratified flows to transition from a turbulent to laminar regime. This laminar regime is not well captured by the large-eddy simulations shown here, limiting the parameter space that we were able to explore. However, we do hope to leverage these simulations to formulate a scale-aware version of the existing melt parameterization on the basis of strong gradients in heat, salt and momentum fluxes with distance from the ice-ocean interface, such that the parameterized melt rate is less dependent on the grid resolution of our coarse models. This improvement would have significant programmatic impact for E3SM as ice-shelf melt fidelity has become more important as we progress toward full coupling between land ice and ocean components.

\section{References}

Abkar, M., Bae, H. J. \& Moin, P. Minimum-dissipation scalar transport model for large-eddy simulation of turbulent flows. Phys. Rev. Fluids 1, 041701 (2016). doi:10.1103/PhysRevFluids.1.041701.

Maronga, B. et al. The Parallelized Large-Eddy Simulation Model (PALM) version 4.0 for atmospheric and oceanic flows: model formulation, recent developments, and future perspectives. Geoscientific Model Development 8, 2515-2551 (2015). doi:10.5194/gmd-8-2515-2015.

\section{Publications}

Begeman, C., Asay-Davis, X., Van Roekel, L. Turbulent boundary layer dynamics below ice shelves from large-eddy simulation. (in preparation for submission to the Journal of Geophysical Research: Oceans).

\section{Financial impact}

The simulations described here were carried out by Co-I Begeman as a part of a Chick Keller Postdoctoral Fellowship awarded by LANL's Center for Space and Earth Science (CSES) The fellowship covered $50 \%$ of Begeman's time with a budget of $\$ 60 \mathrm{k} / \mathrm{year}$. The results of these simulations are expected to feed into parameterizations of ice sheet-ocean interactions being developed in the DOE SciDAC project Probabilistic Sea Level Projections from Ice Sheet and Earth System Models (ProSPect) with an annual budget of \$2.6M (\$1M of which goes to LANL) and the DOE Energy Exascale Earth System Model (E3SM) project, which has an annual budget of $\$ 5.5 \mathrm{M}$. The simulations and associated PALM development also supported the work of the Interdisciplinary Research For Arctic Coastal Environments (InteRFACE) project, with an annual LANL budget of $\$ 350 \mathrm{~K}$. 